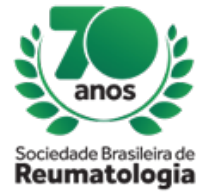

\title{
PERIODIC HYPOKALEMIC PARALYSIS AS THE FIRST CLINICAL MANIFESTATION OF SJOGREN SYNDROME
} IN THE EMERGENCY SECTOR.

Matheus Zanata Brufatto (Faculdade de medicina de Botucatu - UNESP, Botucatu, SP, Brasil), Sean Hideo Shirata Lanças (Faculdade de medicina de Botucatu - UNESP, Botucatu, SP, Brasil), Joao Paulo Canaan (Faculdade de medicina de Botucatu - UNESP, Botucatu, SP, Brasil), Lais De Vico (Faculdade de medicina de Botucatu - UNESP, botucatu, SP, Brasil), Henrique Pereira Sampaio (Faculdade de medicina de Botucatu - UNESP, Botucatu, SP, Brasil), Andrea Almeida Peduti Batista (Faculdade de medicina de Botucatu - UNESP, Botucatu, SP, Brasil), Luiz Eduardo Valente (Faculdade de medicina de Botucatu UNESP, Botucatu, SP, Brasil), Sula Glaucia Lage Pacheco Drumond (Faculdade de medicina de Botucatu UNESP, Botucatu, SP, Brasil), Fabio Vicente Leite (Faculdade de medicina de Botucatu - UNESP, Botucatu, SP, Brasil)

\section{BACKGROUND}

Sjögren's syndrome was described in 1933 with the triad arthritis, dry eye and dry mouth. It's characterized by lymphocyte infiltration in exocrine glands and in other organs, with the production of various autoantibodies. It has several renal manifestations, such as renal tubular acidosis. Periodic Hypokalemic Palisys is a rare manifestation due to renal tubular acidosis.

\section{CASE REPORT}

A 54-year-old female caucasian woman with controlled hypothyroidism initiated fever and hyaline rhinorrhea associated with proximal paresis of the lower and upper limbs. She complained of xerostomia and xerophthalmia of long standing and had ophthalmology diagnosis of keratoconjunctivitis sicca. Serum potassium was $1.6 \mathrm{mmol} / \mathrm{L}$ and creatine phosphokinase was $10.000 \mathrm{U} / \mathrm{L}$, referring to periodic hypokalemic paralysis. She has creatinine ratio of $1.2 \mathrm{mg} / \mathrm{dL}$, no change in blood count, negative inflammatory tests and normal thyroid function. Direct antiglobulin test, lupus anticoagulant, anticardiolipins, anti-dsDNA and anti-SM were all negative. ANA was $1 / 320$ thick dotted nuclear pattern and anti-Ro and anti-La were upper $200 \mathrm{U} / \mathrm{ml}$. She had also rheumatoid factor by latex positive. Chest X-rays and wrists showed no changes. Electrocardiogram showed U-wave. Venous gasometry showed metabolic acidosis with normal anion gap and by associating it with hypokalemia it could be defined Distal Renal Tubular Acidosis attributed to Sjögren's Syndrome. She was treated with hydroelectrolytic support, Prednisone $1 \mathrm{mg} / \mathrm{kg} /$ day and Hydroxychloroquine $400 \mathrm{mg} /$ day, with total resolution of the disease.

\section{CONCLUSION}

We present a case report of Primary Sjögren's Syndrome with rare and severe manifestation diagnosed in an Emergency Unit. Outcome reports on rare diseases with severe manifestations are necessary to enhance therapeutic plans for this kind os patient in the near future. 